\title{
ARTICLE
}

\section{Rapid Detection of Group B Streptococcus (GBS) from artificial urine samples based on IFAST and ATP Bioluminescence Assay: from development to practical challenges during protocol testing \\ in Kenya}

Received 00th January 20xx, Accepted 00th January 20xx DOI: $10.1039 / x 0 x x 00000 x$

\author{
Bongkot Ngamsom, ${ }^{\text {a }}$ Ernest Apondi Wandera, ${ }^{\mathrm{b}}$ Alexander Iles, ${ }^{\text {a }}$ Racheal Kimani, ${ }^{\mathrm{b}}$ Francis Muregi, ${ }^{\mathrm{b}}$ \\ Jesse Gitaka*b and Nicole Pamme*a
}

\begin{abstract}
We report the rapid detection (20 min) of Streptococcus agalactiae, Group B Streptococcus (GBS) employing on-chip magnetic isolation of GBS based on immiscible filtration assisted by surface tension (IFAST), followed by detection of the isolated GBS using an adenosine triphosphate (ATP) bioluminescence assay. Up to $80 \%$ GBS cells were isolated from spiked artificial urine samples with linear responses of bioluminescence signals from isolated cells at $2.3 \times 10^{2}-9.1 \times 10^{5} \mathrm{CFU} \mathrm{mL}^{-1}$, demonstrating great promise for point-of-care detection of pathogenic bacteria in screening urine samples from preg nant women. Practical challenges during initial testing of the developed protocol with urine samples in Kenya are also described.
\end{abstract}

\section{Introduction}

Maternal bacterial infections (MBIs) including Ureaplasma urealyticum, Mycoplasma hominis, Bacteroides spp., Gardnerella vaginalis, Neisseria gonorrhoeae, Chlamydia trachomatis, Treponema pallidum and Group B haemolytic streptococci (GBS) are important contributors to adverse pregnancy and perinatal outcomes such as spontaneous abortions, ectopic pregnancies, still births, premature deliveries, low-birth weight, neonatal mortality and neonatal deaths. ${ }^{1}$ These adverse pregnancy outcomes present a significant global public health challenge with the greatest burden lying in low-income countries in South Asia and subSaharan Africa. ${ }^{2}$ For instance, it is estimated that up to $13 \%$ of deliveries in some African countries are premature, ${ }^{3}$ contributing to about $35 \%$ of neonatal mortality, and it is the leading cause of the under-fives mortality rate. ${ }^{4}$ Premature infants have an increased risk for adverse sequelae including short- and long-term neuro deficits including deafness, blindness and mental retardation as well as other conditions such as diabetes and hypertension. ${ }^{5,6}$

\footnotetext{
a. Department of Chemistry and Biochemistry, Faculty of Science and Engineering University of Hull, Hull, HU6 7RX, UK. E-mail:n.pamme@hull.ac.uk

b. Directorate of Research and Innovation, Mount Kenya University, Thika, KENYA.

E-mail: jgitaka@mku.ac.ke

* Joint senior authors

Electronic Supplementary Information (ESI) available: [details of any supplementary information available should be included here]. See DOI: $10.1039 / x 0 \times x 00000 x$
}

Maternal bacterial infections have indolent sub-clinical courses and are mostly asymptomatic, and hence are poorly diagnosed. A significant proportion of these infections cause chronic subclinical inflammation of the maternal fetal unit compromising the fetal wellbeing, trigger premature labour, delivery and stillbirths. ${ }^{7}$ In spite of this, antenatal care clinics in Kenya, like in other developing countries, have adopted the WHO-recommended syndromic management of MBIs where clinical symptoms are used for diagnosis in absence of laboratory confirmation. ${ }^{8}$ Whilst this approach compensates for the lack of equipment and trained personnel for aetiological diagnosis in such settings, it is deficient in both sensitivity and specificity. Inability to diagnose MBIs and predict the risk of adverse pregnancy outcomes prevents timely interventions that may include available and inexpensive solutions such as use of prenatal steroids, antibiotics or well-planned new-born care. Additionally, existing strategies to test for them are not sensitive enough, do not necessarily indicate current infection, and require sophisticated infrastructure. ${ }^{9}$

Amongst the maternal bacterial infections, GBS has proved to be a major risk for severe neonatal infections, e.g. sepsis, pneumonia and meningitis. ${ }^{10}$ Studies in Kenya have estimated GBS colonisation prevalence in pregnant women to range from $25 \%$ to $31 \%$ with significant association with adverse pregnancy and perinatal outcomes such as stillbirths. ${ }^{11}$ However, managing GBS is problematic because the infections are usually asymptomatic, and thus go undiagnosed until at a later stage when complications develop. Furthermore, in many lowincome countries including Kenya, routine screening for GBS in 
pregnant women does not exist, curtailing preventative care which includes maternal screening via urine culture in the first trimester with antibiotic treatment as needed, followed by rectovaginal culture at 35-37 weeks. Intrapartum antibiotic treatment is indicated for those who test positive at 35-37 weeks. ${ }^{12,13}$ Currently employed laboratory methods for GBS detection are time-consuming cell culturing and low-sensitivity microscopic examination; both of which are not readily available in resource-poor settings. ${ }^{13}$ Polymerase chain reaction $(P C R)$ has the advantages of being faster and generally more sensitive but requires expensive, specialised laboratory instrumentation and highly skilled personnel, which are often not available at more remote and less well-equipped clinics in low-income countries. Hence there is an urgent need for affordable, rapid and accurate point of care screening tests for GBS in low-income areas. Ideally, such a test should be low cost, easy to use, and dispose of, and require only the simplest instrumentation. A suitable test should allow the capturing of pathogens in about $1 \mathrm{~mL}$ of urine or recto-vaginal swab sample, and delivering an easily readable and interpreted result on which an end user can react.

The ATP assay is a highly sensitive detection method based on the reaction between molecular ATP, luciferin and oxygen which is catalysed by the enzyme luciferase. ${ }^{15}$ During the reaction, ATP is changed to AMP, and oxyluciferin and light are produced as by products. The light by-product is directly proportional to the amount of ATP in the analyte. The assay is fast and results can be obtained in less than 5 minutes. The sensitivity of ATP bioluminescence assay in combination with the specificity of immunomagnetic separation (IMS) was first reported by Lee and Deininger, allowing $E$. coli to be detected from beach water within 1 h. ${ }^{15}$ The approach was later applied for $E$. coli detection from food ${ }^{16}$ and wastewater. ${ }^{17}$ However, the approach requires a high volume of sample and reagents, and relies on time-consuming repeat washing associated with IMS, and therefore is not practical for point-of-care diagnosis. In analogy to the conventional IMS, IFAST is a microfluidic technique that allows specific isolation and purification of analytes from matrices in which they are embedded, by means of affinity magnetic separation. ${ }^{18}$ The technique is performed in a microfluidic chip including separate chambers interconnected via gates. Immiscible liquids are allowed to sit next to each other in each chamber, for instance in this case, aqueous sample, olive oil and wash buffer (phosphate buffered saline solution). Functionalised magnetic particles are then added to the sample to capture the analyte of interest. By the application of a movable external magnetic field, only magnetic beads with captured analyte are then directed from the sample chamber, through the oil chamber into the elution buffer chamber, leaving other contaminants in the sample chamber. This allows isolation and purification of analytes to be performed prior to the detection process by a single washing step, using small volumes of reagents. The process is highly specific, fast and can be achieved in seconds or a few minutes. IFAST has been applied for concentration of nucleic acids from cell lysates, ${ }^{19}$ breast cancer cells from whole blood, ${ }^{18}$ RNA from HIV spiked serum, ${ }^{20}$ and Helicobacter pylori from stool samples. ${ }^{21}$
The combination of the IFAST specificity and ATP assay sensitivity has been successfully exploited for rapid detection of E. coli 0157: $\mathrm{H7}$ from contaminated water. ${ }^{22}$ Here, we explored the concept for GBS detection from urine samples (Fig. 1), for its suitability for point-of-care rapid screening of GBS colonisation in pregnant women in rural areas of Kenya enabling appropriate and timely clinical action.
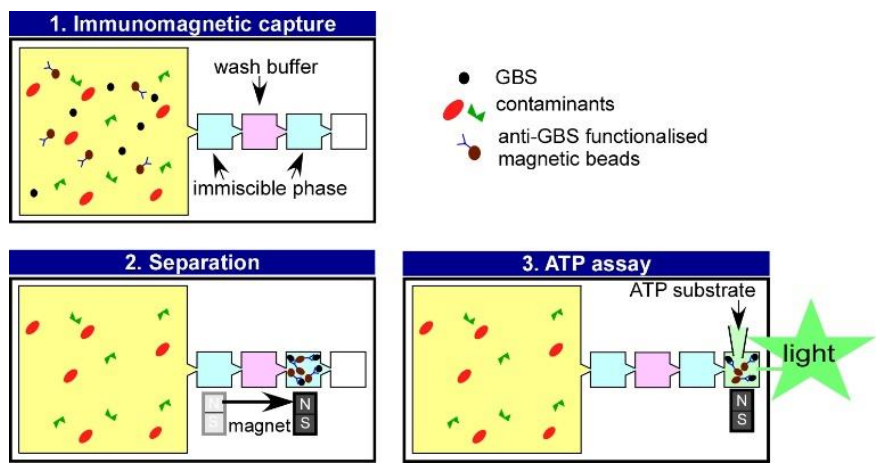

Fig.1 Concept for microfluidic isolation and detection of GBS by IFAST/ATP assay, comprising of three steps: (a) immunomagnetic binding of magnetic particles to GBS in the sample chamber by agitation; (b) separation, whereby bead-captured GBS cells are magnetically isolated from contaminants through wash chambers; (c) detection whereby lysing agents and ATP substrate are loaded into detection chamber with light being produced upon ATP reaction.

\section{Experimental}

\section{Ethical approval}

This study was approved by the Mount Kenya University Ethical Review Committee (MKU/ERC/0765). Urine samples were obtained from Mount Kenya University, Department of Clinical Services. Written informed consent was obtained from all the study participants after the nature and possible consequences of the study had been fully explained to them.

\section{Microfluidic chip}

The IFAST microfluidic chip (Fig. 2) featured a large sample chamber (26 $\mathrm{mm}$ wide, $26 \mathrm{~mm}$ long, $4 \mathrm{~mm}$ deep), three wash chambers and a wash chamber ( $3 \mathrm{~mm}$ wide, $3 \mathrm{~mm}$ long, $4 \mathrm{~mm}$ deep) interconnected via trapezoidal microfluidic gates ( $3 \mathrm{~mm}$ to $500 \mu \mathrm{m}$ wide, $1.5 \mathrm{~mm}$ long, $250 \mu \mathrm{m}$ deep). An inverted replica mould was milled via CNC (Datron M7, Germany) into PMMA. IFAST devices were then fabricated from polydimethylsiloxane (PDMS, Sylgard 184, Dow Corning) via soft lithography. PDMS monomer was mixed with the curing agent at a volume ratio of 10:1. The mixture was degassed under vacuum for $30 \mathrm{~min}$ and poured onto the master mould and cured for $1 \mathrm{~h}$ at $60{ }^{\circ} \mathrm{C}$. The chips were sealed at the bottom with optical adhesive film (Thermo Scientific, USA) prior to use. The microfluidic chip employed for these IFAST experiments incorporated the sample chamber with small access holes. After sample loading, these holes were sealed with optical adhesive film which helped to prevent an operator from coming into contact with pathogencontaminated samples; an improved feature from the previously reported IFAST chip. ${ }^{22}$ 
(a)

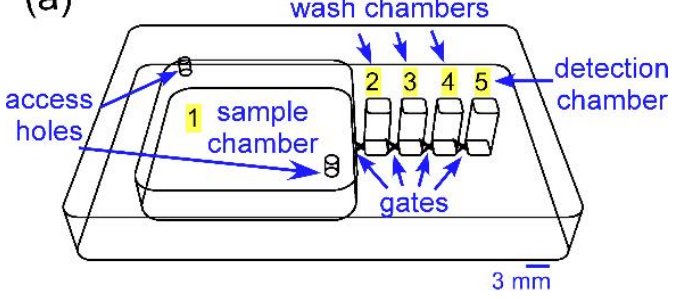

(b)

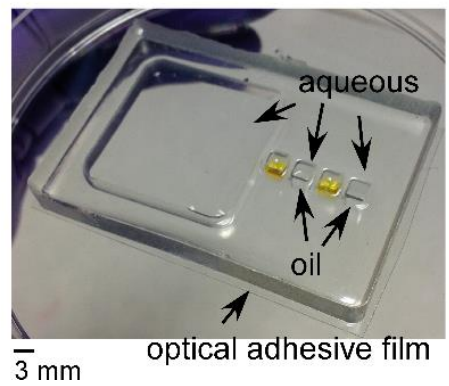

Fig. 2 (a) Schematic of the device featuring the five chambers and gates. (b) Photograph of the PDMS chip with adhesive tape bottom.

\section{Detection system}

Detection of luminescence from on-chip ATP assays was achieved by placing the microfluidic device on a holder directly above the photomultiplier tube (PMT) element (H5784, Hamamatsu Photonics, Enfield, UK) of the custom-made lighttight steel box $(168 \mathrm{~mm} \times 140 \mathrm{~mm} \times 90 \mathrm{~mm}$ ) with a protective shutter. ${ }^{23}$ The PMT active area was $8 \mathrm{~mm}$ in diameter, providing a spectral response of $300-650 \mathrm{~nm}$, with a peak wavelength of $420 \mathrm{~nm}$. The PMT was supplied with $\pm 5 \mathrm{~V}$ for operation. Bioluminescence signals from ATP assays were displayed via a digital multi-meter connected to the output of the detection box (Fig. 3).

(a)

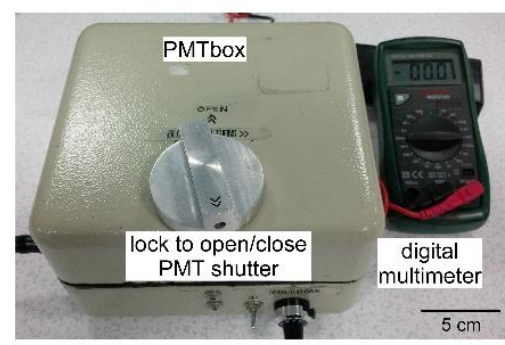

(b)

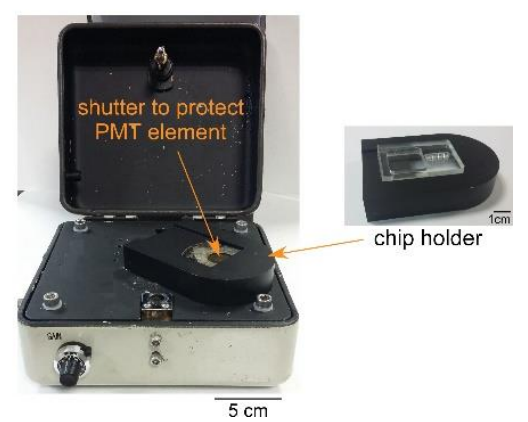

Fig. 3 (a) The portable device containing a PMT element for luminescence detection, connected to a digital multi-meter for readout. (b) View inside the detection box showing the chip holder located above the PMT and the protective shutter.
Preparation of functionalised magnetic beads specific to GBS (antiGBS magnetic beads)

The biotinylated-rabbit polyclonal antibody against GBS (AB19983, Abcam) was conjugated to streptavidin-M280 Dynabeads (11205, Invitrogen) via streptavidin-biotin binding. Twenty-five microlitres of streptavidin-M280 suspension (10 $\mathrm{mg}$, ca. 6-7 $\times 10^{8}$ magnetic beads per $\mathrm{mL}$ ) was taken from the stock suspension and washed with phosphate buffered saline solution containing Tween20 ( $1 \mathrm{~mL}$ PBST: $0.01 \mathrm{w} / \mathrm{v} \%$ Tween 20). To the washed bead suspension in PBST, was added the antibody against GBS to afford a final concentration of $10 \mu \mathrm{g}$ antibody per $1 \mathrm{mg}$ magnetic beads. The suspension was incubated at room temperature $\left(22{ }^{\circ} \mathrm{C}\right)$ for $30 \mathrm{~min}$ using a rotator (Stuart, UK) set at $30 \mathrm{rpm}$. The unbound antibody was washed three times in phosphate buffer saline with $0.1 \mathrm{w} / \mathrm{v} \%$ bovine serum albumin (BSA) to prevent non-specific binding, and re-suspended in PBST (similar to the original volume taken from the bead stock suspension. Verification of binding between antibody against GBS was performed by incubating GBS cell suspension in PBST $(1 \mathrm{~mL})$ with $10 \mu \mathrm{L}$ fluorescein isothiocyanate labelled antibody against rabbit IgG $\left(1 \mathrm{mg} \mathrm{mL}^{-1}\right.$, FITC-anti-rabbit IgG, 81-6111, Invitrogen) at room temperature for $30 \mathrm{~min}$ employing a rotator set at $40 \mathrm{rpm}$. The unbound FITCanti-rabbit IgG was washed ( $3 \times 1 \mathrm{~mL}$ PBST) by centrifugation (Eppendorf Minispin, UK) at $5000 \mathrm{rpm}$ for $5 \mathrm{~min}$. The antibody bound cells were visualised under a fluorescence microscope (Nikon Eclipse, TE2000-U, UK).

\section{Spiked artificial urine samples}

\section{Preparation of artificial urine samples spiked with GBS}

A single colony of GBS (NCTC 8181, Pro-lab Diagnostics, UK) grown on blood agar was inoculated in $10 \mathrm{~mL}$ brain heart infusion broth (Oxoid, UK) and incubated overnight at $37^{\circ} \mathrm{C}$. The overnight culture was subjected to centrifugation (VWR Megastar 1.6, UK) at 5,000 rpm for $4 \mathrm{~min}$ and the media was removed. The cells were washed with PBST $(2 \times 10 \mathrm{~mL})$ and re-suspended in PBST to obtain a reading of 0.25 absorbance units from the spectrophotometer measured at $625 \mathrm{~nm}$ (CECIL 1011 spectrometer, 1000 Series); corresponding to ca. $10^{8} \mathrm{CFU}$ $\mathrm{mL}^{-1}$. A series of decimal dilutions were prepared ( $d-1$ to $d-5$ corresponding to $\mathrm{ca} .10^{7}$ to $10^{3} \mathrm{CFU} \mathrm{mL}^{-1}$. Subsequently, $0.1 \mathrm{~mL}$ of d-5 was plated in duplicate on blood agar and incubated overnight at $37{ }^{\circ} \mathrm{C}$ in order to confirm the actual GBS concentration in PBS. The number of GBS cells present was calculated by counting colonies on the plates $(\times 2)$ taking into account the dilution factor. An appropriate concentration of GBS $\left(10^{3}-10^{5} \mathrm{CFU} \mathrm{\textrm {mL } ^ { - 1 }}\right)$ was spiked into artificial urine (SigmaAldrich, UK) containing $0.001 \%(\mathrm{w} / \mathrm{v})$ Tween 20 (PBST) for IFAST experiments.

\section{Off-chip immunomagnetic separation (IMS) of GBS from spiked samples}

To one millilitre of GBS spiked samples (of each concentration), were added $20 \mu \mathrm{L}$ of anti-GBS functionalised magnetic beads and the mixture was allowed to incubate at room temperature 
for 10 - 30 min using a rotator (Stuart, UK) set at $40 \mathrm{rpm}$. An external NdFeB magnet (Magnet Sales, UK) was attached to the side of the sample tube for $2 \mathrm{~min}$ to separate the GBS-bound magnetic beads. The remaining supernatant $(0.1 \mathrm{~mL})$ was removed (while still holding the magnet to the tube) and serially diluted in PBS until an approximated $10^{3} \mathrm{CFU} \mathrm{mL} \mathrm{m}^{-1}$ dilution was obtained. A volume of the $10^{3} \mathrm{CFU} \mathrm{mL}^{-1}$ dilution $(0.1 \mathrm{~mL})$ was plated on blood agar plates ( $\times 2$ for each spiked buffer sample) and incubated overnight at $37{ }^{\circ} \mathrm{C}$. The resulting colonies were enumerated and the percentage pathogen captured by IMS was calculated by the difference in the colony counts in the samples before and after the IMS process. After removing the supernatant, the GBS-bound beads were washed with $1 \mathrm{~mL}$ PBST (0.01\% Tween 20) three times and re-suspended in $0.1 \mathrm{~mL}$ PBST (0.01\% Tween 20). The viability of the isolated cells was investigated by plating $0.1 \mathrm{~mL}$ of the $10^{3} \mathrm{CFU} \mathrm{mL}-1$ dilution of the re-suspended beads on blood agar plates $(\times 2$ for each spiked buffer sample) and incubated overnight at $37^{\circ} \mathrm{C}$.

\section{IFAST}

\section{Chip Loading}

Loading of the IFAST chip was carried out by firstly filling the sample chamber with $1 \mathrm{~mL}$ GBS spiked artificial urine sample containing $0.001 \%$ Tween 20 (PBST, 0.001\% Tween 20). Next, chambers 3 and 5 were filled with $20 \mu \mathrm{L}$ PBS containing $0.01 \%$ Tween 20 (PBST, 0.01\% Tween 20). Then olive oil (SigmaAldrich, USA) was loaded into chambers 2 and 4 . Finally, $25 \mu \mathrm{L}$ anti-GBS functionalised magnetic beads were mixed with the spiked sample in chamber 1 .

\section{Immunomagnetic separation of GBS}

Immunomagnetic binding (5-20 min, incubation at room temperature) was performed by manual agitation of the IFAST chip. The captured GBS was subsequently aggregated inside the sample chamber employing a magnet assembly (1 $\mathrm{min})$. By horizontally moving the magnet assembly across the length of the IFAST chip, the immunomagnetic beads carrying GBS cells were transferred from the sample chamber and purified through the immiscible liquid wash chambers (2-4), and finally stored in buffer in chamber 5 . The isolation performance and viability of the isolated cells were quantified by plating the supernatant from chamber 1 , and the cell-bound beads from chamber 5 , respectively.

\section{Specificity test}

Two strains of E. coli (NCTC 12241 and NCTC 12900), Staphylococcus aureus (S. aureus, NCTC 12023) and Neisseria gonorrhoeae (N. gonorrhoeae, NCTC 8375) were purchased from Pro-lab Diagnostics, UK. Differential agars for GBS (GBSbrilliance), S. aureus (Staph-Brilliance) and N. gonorrhoeae (Chocolate GC selective agar) were supplied by Oxoid, UK. Single colonies of $E$. coli and $S$. aureus were separately inoculated in tryptic soy broth (TSB, Oxoid, UK), whilst brain heart infusion broth was utilised for GBS; all inoculations were performed overnight at $37^{\circ} \mathrm{C}$. The overnight culture of E. coli and S. aureus was prepared similarly to that of GBS. Bacteria at $c a .10^{3} \mathrm{CFU}$ $\mathrm{mL}^{-1}$ were spiked into artificial urine sample containing $0.01 \mathrm{w} / \mathrm{v}$
Tween 20. For $N$. gonorrhoeae spiked sample, inoculation was carried out in brain heart infusion broth and incubated at $35^{\circ} \mathrm{C}$ under $\mathrm{CO}_{2}$ atmosphere for $3 \mathrm{~h}\left(\mathrm{OD}_{540}=0.550\right.$; corresponding to ca. $10^{8} \mathrm{CFU} \mathrm{mL}-1$ ). Opting the similar enumeration process for GBS, N. gonorrhoeae was spiked to artificial urine $\left(10^{3} \mathrm{CFU}\right.$ $\left.\mathrm{mL}^{-1}\right)$. Anti-GBS conjugated magnetic beads ( $0.25 \mathrm{mg}$ beads) were subjected to $1 \mathrm{~mL}$ of artificial urine samples spiked with GBS, S. aureus, two strains of E. coli, and N. gonorrhoeae. Immunomagnetic binding was performed in the sample chamber of the IFAST chip; incubation was achieved by $30 \mathrm{~min}$ manual agitation. Following magnetic separation, the supernatant from each sample was plated on blood agar as well as differential agar and compared with colonies from the original sample (control) for quantification of capture efficiency.

\section{ATP assays}

Twenty microlitres of ATP standard solution or bacterial sample were mixed with $20 \mu \mathrm{L}$ ATP assay substrate (Bactiter Glo, Promega, USA) in the detection chamber (chamber 5) of the IFAST chip. Next, the chip was placed on the chip holder above the PMT element of the detection box and the box was shut. The maximum detected luminescence, displayed as voltage for each sample was recorded. A calibration curve was created based on a base 10 logarithmic plot of the detected luminescence (relative light unit) versus the concentration of the ATP standard (or bacterial cell).

\section{On-chip IFAST/ATP assays}

The chip loading and IFAST isolation and purification procedure was similar to that described previously, ${ }^{22}$ except that after immunomagnetic binding in the sample chamber, followed by washing through a series of oil and wash buffer (chamber 2-4), the GBS-bound beads were temporarily stored in chamber 4 whist supernatant from the sample chamber was collected for further analysis of isolation efficiency by plating. Next, chamber 5 was loaded with ATP assay substrate and the beads with isolated GBS were moved from chamber 4 to chamber 5 . The beads in chamber 5 were moved up and down through the liquid within the chamber by alternating the positions of the magnet assembly from below the chamber to above the chamber for ca. $20 \mathrm{~s}$. This was to ensure adequate dispersion of the beads to facilitate the ATP assay. Subsequently, the chip was secured on the chip holder above the PMT element of the detection box and the box was shut. The maximum voltage displayed from each concentration was recorded and subtracted from the blank test (negative control = artificial sample without GBS). Average logarithmic values of voltage and cell concentration were plotted and analysed from three independent experiments $(n=3)$.

\section{Spiked patient's urine samples}

An ATCC strain of S. agalactiae, a kind donation from the Kenya Medical Research Institute (KEMRI)-Walter Reed Research Program, was revived on blood agar and incubated in a $37{ }^{\circ} \mathrm{C}$ incubator inside a candle jar to supply the culture with $\mathrm{CO}_{2}$ (there was no $\mathrm{CO}_{2}$-incubator at our facility). Following a 48-hour incubation, the colonies were sub-cultured in Tryptic Soy Broth 
(TSB) and incubated at $37^{\circ} \mathrm{C}$ for $4-5$ hours. The optical density (OD) of the inoculum was checked using a spectrophotometer

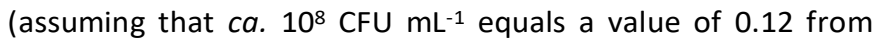
OD540). Serial dilutions of the inoculum were prepared in PBS from $10^{7}-10^{3} \mathrm{CFU} \mathrm{mL}-1$. To prepare a $10^{3} \mathrm{CFU} \mathrm{mL} \mathrm{m}^{-1} \mathrm{GBS}$ spiked urine sample, a volume of $0.1 \mathrm{~mL}$ was taken from $10^{5} \mathrm{CFU} \mathrm{mL}^{-1}$ GBS dilution and made up to $10 \mathrm{~mL}$ with urine sample $[0.1 \mathrm{~mL} x$ $10^{5} \mathrm{CFU} \mathrm{mL}^{-1} / 10 \mathrm{~mL}=10^{3} \mathrm{CFU} \mathrm{mL}^{-1}$ ). This was meant to reduce TSB in the spiked urine sample, preventing further growth over time. GBS was spiked into an archived urine sample (Mount Kenya University, Thika, Kenya). The sample was divided into two portions. The first portion was used as a positive control and was labelled as 'crude'. The other portion was filtered through a $0.22 \mu \mathrm{m}$ membrane and labelled as 'filtered', a representation of negative control as the bacteria should be retained within the membrane. Standard inoculum of GBS was prepared in a similar manner as GBS prepared for spiked artificial samples. Both samples were spiked with GBS at different concentrations.

\section{Results and discussion}

Functionalised magnetic beads for immunomagnetic capture of GBS

On-chip isolation of GBS from artificial urine samples in the present communication exploited magnetic particles functionalised with an antibody against GBS. In our previous work, the anti-E. coli 0157 magnetic beads were commercially available and have been extensively utilised for isolation of E. coli. ${ }^{22}$ However, anti-GBS conjugated magnetic beads are not commercially available, and were therefore prepared in-house, employing a non-covalent, and yet stable, streptavidin-biotin bonding. ${ }^{24}$ Binding of the commercially available biotinylated antibody against GBS (anti-GBS, Abcam) to GBS cells was first validated utilising a fluorescently-labelled secondary antibody, binding the anti-GBS antibody to GBS cells (Step 1, Fig. S1 and Fig. S2a). By incubating the commercially available streptavidinlabelled magnetic beads (Dynabeads) with biotinylated antibody against GBS (anti-GBS) at room temperature for $30 \mathrm{~min}$, functionalised magnetic beads capable of binding to GBS cells were obtained. The fluorescent beads upon the addition of the same fluorescently-labelled secondary antibody also qualitatively confirmed the streptavidin-biotin bonding (Fig. S2b), demonstrating a successful preparation of anti-GBS conjugated magnetic beads (Step 2, Fig. S1).

The ability to isolate GBS from spiked buffer (PBS) by the prepared functionalised magnetic beads (step 3, Fig S1) was next quantified. Taking into consideration that testing of the protocol was aimed for a resource-limited laboratory of Kenya, immunomagnetic capture was investigated at room temperature as well as at $4{ }^{\circ} \mathrm{C}$ (Fig. S3). Higher capture efficiency was observed at room temperature as a result of accelerated interaction between antibody and GBS cells with increasing temperature. $\mathrm{Ca}$. $85 \%$ immunomagnetic binding was observed from off-chip tube-based conjugation of anti-GBS antibody to magnetic beads via streptavidin-biotin reaction at room temperature utilising $50 \mu \mathrm{g}$ antibody per mg beads.
The specificity of our protocol for GBS isolation depends on the specificity of the antibody functionalised magnetic beads. Although non-specific binding to other non-tested bacteria cannot be ensured, the specificity of the prepared anti-GBS conjugated magnetic beads was verified against most common bacteria found in infected urine samples, keeping in mind that the developed protocol was aimed towards testing of urine samples from pregnant women. This testing included E. coli, the most common cause for urinary tract infection. ${ }^{24}$ Uncommonly associated with urinary tract bacteria in the general population, $S$. aureus can cause ascending urinary tract colonisation and infection in certain patients. ${ }^{26} \mathrm{~S}$. aureus was also reported as the second most isolated bacteria in pregnant women in Nigeria. ${ }^{26}$ Additionally, screening was performed with $N$. gonorrhoeae, one of the pathogenic bacteria causing sexual transmitted diseases which can be present in urine. ${ }^{27}$ The functionalised magnetic beads were specific only to GBS and did not bind to other organisms tested (Table 1).

Table 1 Specificity test of the anti-GBS immunomagnetic beads against GBS, S. aureus, E. coli and N. gonorrhoeae

\begin{tabular}{lc}
\hline Organism & $\begin{array}{c}\text { Binding to anti-GBS conjugated } \\
\text { magnetic beads }\end{array}$ \\
\hline GBS & + \\
S. aureus & - \\
E. coli O157 & - \\
E. coli (NCTC 12241) & - \\
N. gonorrhoeae & - \\
\hline
\end{tabular}

+ confirmed binding, - no binding

\section{On-chip immunomagnetic separation by IFAST (on-chip IMS)}

Purification of the isolated bacterial cells by on-chip IFAST relies on a single traverse of bacterial-bound magnetic particles through a series of microscale virtual walls created by surface tension between immiscible liquids, by a handheld external magnet. The immiscible liquid chosen for IFAST experiments was olive oil due to its high interfacial energy with the aqueous phase, ${ }^{18}$ and its easy accessibility. Microscopic observation demonstrated immiscible liquids laying side-by-side each other, creating virtual walls from aqueous/oil interfaces between the gates connecting the chambers in which the immiscible liquids were contained (Fig. 4a). The pressure created by the movement of magnetic beads through aqueous/hydrophobic regions slightly disturbed, but did not destroy, these interfaces (Fig. 4b). This allowed passage of beads through to the next chamber without carry-over of liquid from the previous chamber. By repeating a series of hydrophobic/hydrophilic chambers (wash chambers), purification of immunomagnetic beads can be achieved in an IFAST chip within seconds, a muchsimplified process compared to the conventional tube-based immunomagnetic separation. 


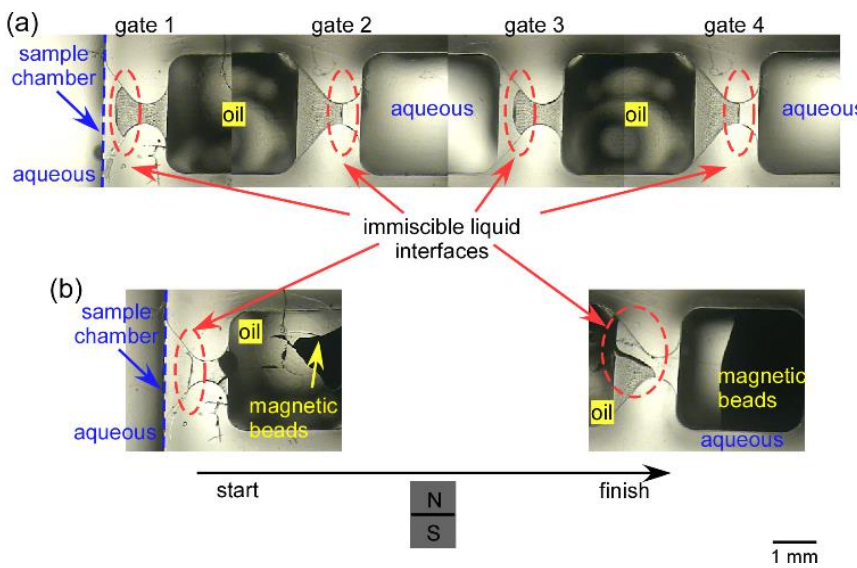

Fig. 4 Microscopic images of immiscible liquid interfaces: (a) between chambers containing aqueous and oil samples, (b) interfaces through which magnetic beads were munipulated.

Evaluation of the IFAST chip for the concentration and purification of GBS employing immunomagnetic beads was next carried out. Immunomagnetic binding (Step 1, Fig. 1) was achieved by manual agitation of the IFAST chip to facilitate mixing between the anti-GBS functionalised beads and the GBS cells. This method does not only provide good capture (ca. $90 \%$ efficiency in $15 \mathrm{~min}$, Fig. 5), but it also eliminates the requirement for external power associated with an automated setup, making it suitable for resource-constrained settings. Purification of the isolated GBS through the immiscible liquid wash chambers (Step 2, Fig. 1) showed uncompromised viability of the captured GBS cells by short exposure to olive oil (confirmed by plating of the magnetic beads after purification through wash chambers, results not shown here). This process took less than $1 \mathrm{~min}$, and required fewer reagents as compared to conventional tube-based IMS.

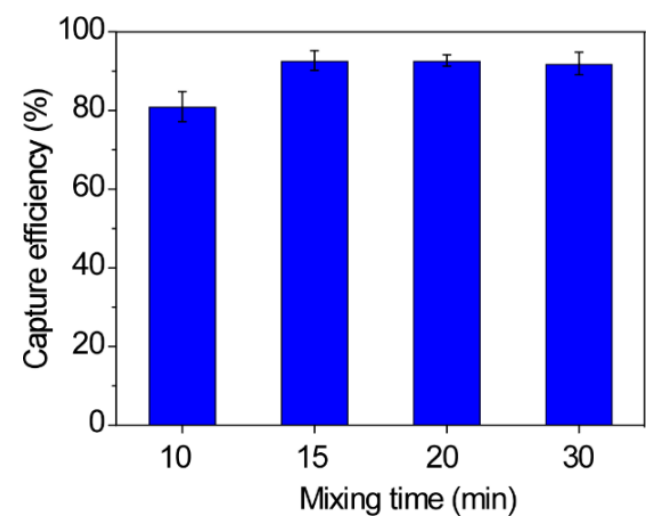

Fig. 5 Effect of incubation time on immunomagnetic capture of GBS from spiked artificial urine samples performed in IFAST chips. (200 CFU mL-1, $n=3$ ).

\section{On-chip ATP assays for GBS detection}

Detection of isolated GBS in the following step of the design approach (Step 3, Fig. 1) exploited the bioluminescence reaction between molecular ATP from the isolated cells and luciferin/luciferase. The sensitivity of the assay allows fast detection and results to be obtained within 5 min, utilising the same set-up as described in our previous communication. ${ }^{22}$ The portable PMT detection system showed comparable results to a commercial luminometer for E. coli 0157.

Reproducibility of the on-chip ATP assays utilising the PMT detection system was first investigated. ATP standard solutions at various concentrations were loaded in the detection chamber of the IFAST chip, into which the ATP assay substrate was added and the chip placed inside the portable PMT detection box for reading. Luminescence produced by ATP standard solutions were directly proportional to ATP concentrations at 0.01 $10 \mathrm{nM}$ with standard deviation $\leq 0.14$ (Fig. 6a). Next, the on-chip ATP assays were performed on $10^{5}-10^{8} \mathrm{CFU} \mathrm{mL}-1$ GBS whole cells (Fig. 6b). ATP levels $\left(3.43 \times 10^{-11} \mathrm{M}\right)$ corresponding to $1 \mathrm{CFU}$ of GBS per $\mathrm{mL}$ can be detected with reagents and equipment used in the present bioluminescent assay. By integrating onchip detection based on ATP assays and developing a portable detection system, quantification of isolated GBS can be performed in two simple consecutive steps. This eliminates practical errors from sample handling as well as the need for a downstream detection instrument, enabling a less expensive, easy-to-operate, and yet sensitive platform that is suitable for point-of-care diagnosis. Patients can provide a sample and wait less than $20 \mathrm{~min}$ to obtain results.
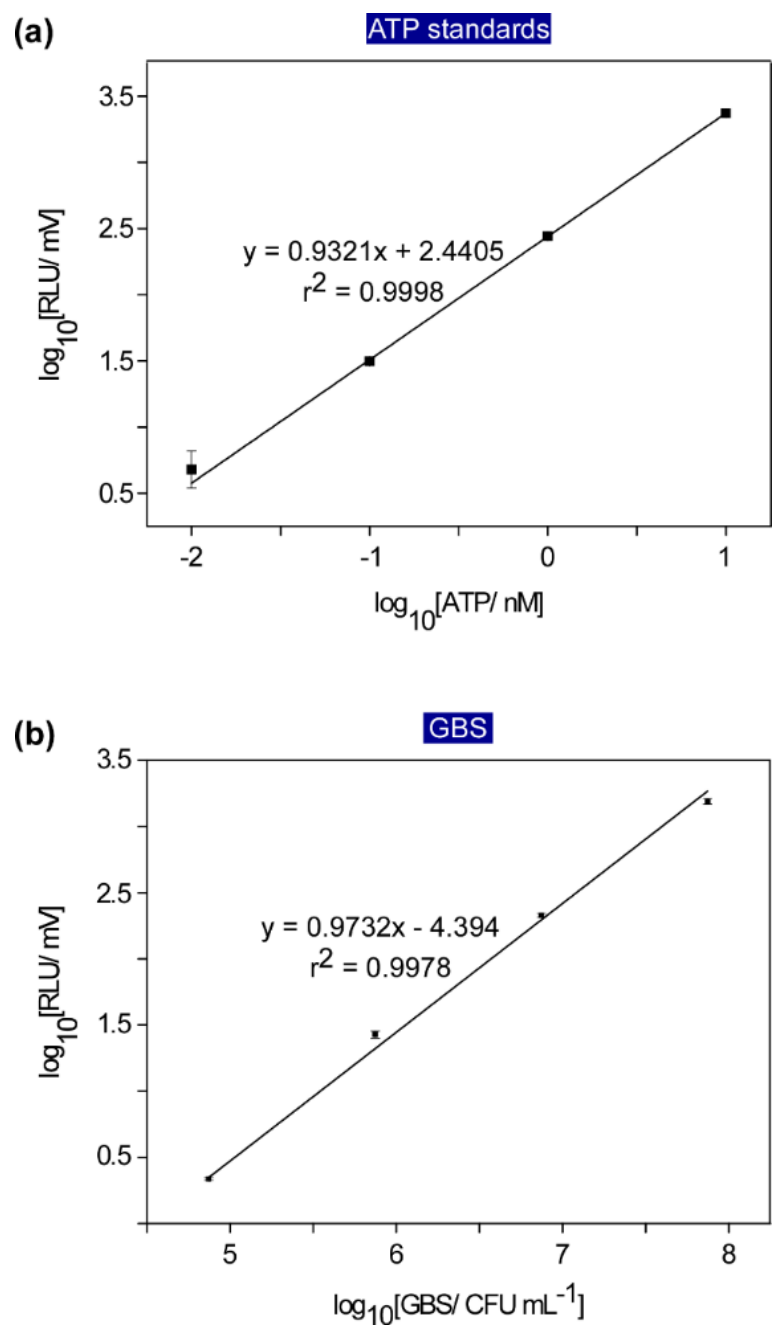

Fig. 6 Luminescence signals observed from on-chip ATP assays using the PMT detection box for (a) ATP standards and (b) GBS whole cells, respectively $(n=3)$. 
On-chip IFAST/ATP assays for rapid detection of GBS from artificial urine samples

Having demonstrated the individual steps of on-chip immunomagnetic separation (IFAST- steps 1 and 2, Fig. 1), and on-chip ATP assays (step 3, Fig. 1), the combined on-chip IFAST/ATP assays was next investigated with GBS spiked artificial samples. Although lower capture efficiency of the functionalised magnetic particles was observed with increasing GBS concentration and will require further optimisation (Fig. 7a), a good linearity $\left(r^{2}=0.9927\right.$, s.d. $\left.<1 \%\right)$ was obtained from a logarithmic plot between concentrations of isolated GBS cells $\left(2.30 \times 10^{2}-9.1 \times 10^{5} \mathrm{CFU} \mathrm{mL}^{-1}\right)$ and detected bioluminescence responses from the ATP assays in the following step (Fig. 7b). The entire process took less than $20 \mathrm{~min}$ (ca. $16 \mathrm{~min}$ for immunomagnetic isolation and purification of GBS from spiked artificial urine samples, and $\leq 2 \mathrm{~min}$ for detection of isolated cells). The present set-up could detect $230 \mathrm{CFU}$ of GBS from $1 \mathrm{~mL}$ spiked artificial urine sample. With improved immunomagnetic capture, the system should allow lower detection levels (6 CFU E. coli 0157 were detected from $1 \mathrm{~mL}$ spiked PBS sample in our previous communication). ${ }^{22}$
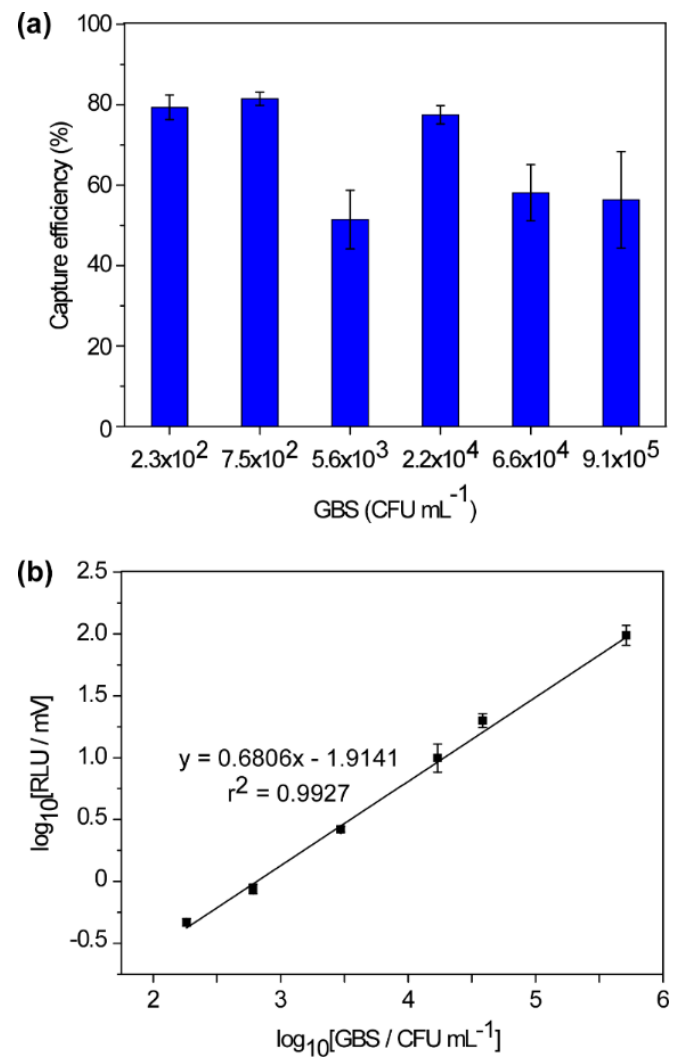

Fig. 7 On-chip IFAST/ATP assays for rapid detection of GBS from spiked artificial urine samples employing the developed microfluidic system and functionalised magnetic particles. (a) Isolation performance $(n=3)$. (b) Excellent linearity obtained from bioluminescence responses of isolated GBS cells $(n=3$, s.d. $<1 \%$ ).

On-chip IFAST/ATP assays for rapid detection of GBS in spiked patient's urine samples

The successful development of a microfluidic approach based on IFAST/ATP assays was next tested with real patient urine samples. The results of the IFAST/ATP assays of patient urine samples are summarised in Table 2. An average of 8 CFU was observed from duplicate plating of filtered sample without GBS (blank), suggesting that the majority of background organisms was indeed retained on the membrane. On the other hand, there was a vast number of colonies observed from unfiltered crude sample without spiked GBS (blank). It was not possible to distinguish GBS colonies from background microorganisms without differential agar, hence no capture efficiency was quantified from GBS spiked-crude samples. As for the subsequent step of ATP assay, non-specific binding of the background microflora to the immunomagnetic beads was apparent, indicated by the much-increased luminescence signal from the blank crude sample with respect to that from the blank filtered sample. The unambiguous increases in luminescence

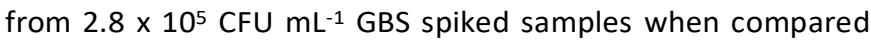
with blanks from both filtered and crude samples validated the IFAST/ATP assay approach for rapid isolation and detection of GBS from real urine samples. However, more investigation is required to (1) increase capture efficiency, (2) reduce nonspecific binding, enabling the approach to detect lower concentration of GBS without false positives. Increasing the BSA concentration (up to $1 \%$ ) may help washing off unbound antibody and reduce non-specific binding. The total concentration of Tween 20 in the sample chamber can be investigated to allow less carry-over background microorganisms without the liquid interface being compromised. Developing a chip design with more wash stages or longer chambers can also be investigated to improve purification.

Table 2. Results of IFAST/ATP assays performed on patient's urine samples.

\begin{tabular}{ccccc} 
GBS & \multicolumn{2}{c}{ Filtered } & \multicolumn{2}{c}{ Crude } \\
\cline { 2 - 5 }$\left(\right.$ CFU mL $\left.{ }^{-1}\right)$ & $\begin{array}{c}\text { Capture } \\
\text { efficiency } \\
(\%)\end{array}$ & $\begin{array}{c}\text { Luminescence } \\
(\mathrm{mV})\end{array}$ & $\begin{array}{c}\text { Capture } \\
\text { efficiency } \\
(\%)\end{array}$ & $\begin{array}{c}\text { Luminescence } \\
(\mathrm{mV})\end{array}$ \\
\hline 0 (blank) & - & 39.1 & $\mathrm{~N} / \mathrm{A}$ & 80.9 \\
$3.3 \times 10^{2}$ & 38.5 & 40.0 & $\mathrm{~N} / \mathrm{A}$ & 81.2 \\
$1.4 \times 10^{3}$ & 0 & 39.9 & N/A & 128.4 \\
$2.3 \times 10^{4}$ & 0 & 40.4 & N/A & 130.1 \\
$2.8 \times 10^{5}$ & 58.6 & 43.2 & N/A & 168.7 \\
\hline
\end{tabular}

Ultimately, the on-chip IFAST/ATP assay was aimed at point-ofcare testing of GBS colonisation from urine samples of pregnant women in rural areas of Kenya. The fast, and reproducible, results obtained from the developed platform should ideally enable timely clinical actions to be made accordingly. However, some challenges have risen during initial tests performed at Mount Kenya University, Kenya; the major barriers being the lengthy process in obtaining reagents. All reagents, including magnetic particles, antibody and ATP assay substrates had to be shipped to Kenya. Delays in clearance of reagents at the point of entry can adversely affect their functioning, especially, the antibody and ATP assay substrates. This may partly explain the low capture efficiency observed for the detection of GBS in 
spiked patient urine samples by IFAST/ATP assays. Thus, the success of the developed protocol/device relies greatly on effective procurement. Replacing the temperature-sensitive antibody with more robust aptamers is a possible solution to this problem.

\section{Conclusions}

A simple and fast system for GBS detection from urine samples has been explored for the first time using IFAST/ATP assays. Isolation and assays were combined in one chip, and results were obtained within $20 \mathrm{~min}$. The developed device showed some potential when implemented with real patient urine samples, although it was limited by sensitivity to background microflora. Overcoming this disadvantage will be the focus of future investigations. Once fully developed, the microfluidic IFAST/ATP assay approach could be implemented for point-ofcare diagnosis of pathogenic bacteria.

\section{Conflicts of interest}

There are no conflicts to declare.

\section{Acknowledgements}

This work was financially supported by an Institutional Links grant under the Newton Utafiti Fund partnership between the UK and Kenya. Prof. G.M. Greenway and Mr. N. Parkin are gratefully acknowledged for their kind support on the detection system. Technical assistants from Mr C. Lloyd and Ms. M.W. Njeri are greatly appreciable.

\section{References}

1 V. Agrawal and E. Hirsch, Semin Fetal Neonatal Med., 2012,17,12;

2 H. Blencowe, S. Cousens, M.Z., Oestergaard, C.D., Moller A.B., Narwal R., Lancet, 2012, 379, 2162.

3 S. Chawanpaiboon, J.P. Vogel, A.B. Moller, P. Lumbiganon, M. Petzold, D. Hogan, Lancet, 2019, 7, e37.

4 M.S., Harrison and R.L., Goldenberg, Semin Fetal Neonatal Med.., 2016, 21(2), 74 - 79

5 R.M. Patel, S. Kandefer, M.C. Walsh, E.F. Bell, W.A Carlo, A.R. Laptook, P.J. Sánchez, S. Shankaran, K.P. Van Meurs, M.B. Bal, E.C. Hale, N.S. Newman, A. Das, R.D. Higgins, B.J. Stoll. N Engl J Med. 2015 Jan 22;372(4):331-40.

6 M. Bertagnolli, T.M. Luu, A.J. Lewandowski, P. Leeson, A.M. Nuyt. Curr Hypertens Rep. 2016;18(4):28

7 M. Cappellettl, S. Della Bella, E. Ferrazzi, D. Mavilio, S.J. Divanovic. Leukoc Biol. 2016;99(1):67-78.

8 www.emro.who.int/aiecf/web79.pdf

9 E.M. Catherine, A.C. Smith, M.E. Natoli, K. A. Kundrod, R. Richards-Kortum. Lab Chip. 2017 Oct 11; 17(20): 3351-3387.

10 B.R. Berg, J.L. Houseman, M.A. Garrasi, C.L. Young and D.W. Newton, J. Clin. Microbiol., 2013, 51, 1253.

11 E.Were, Esamal F, Liechty E, et al. Annual Kenya Obstetrical and Gynaecological Society (KOGS) meeting, 2006

12 S. Schrag S., Clin. Infect. Dis., 2004, 39, 1136.
13 G. Di Renzo, P. Melin, A. Berardi, M. Blennow, X. Carbonell-Estrany, G. Donzelli, J. Matern.-Fetal Neonatal Med., 2015; 28, 766.

14 M. Rosa-Fraile and B. Spellerberg, J. Clin. Microbiol., 2017, 55, 2590.

15 J. Lee and R.A. Deininger, Luminescence, 2004, 19, 31.

16 S.-I. Tu, D. Patterson, J. Uknalis, and P.Irwin, Food Res. Inter., 2000, 33, 375.

17 R.N. Bushon, C.A. Likirdopulos and A.M. Brady, Water Res. 2009, 43, 4940.

18 S.M. Berry, L.N. Strotman, J.D. Kueck, E.T. Alarid, and D.J. Beebe, Biomed. Microdevices, 2011, 13, 1033; S.M. Berry, K.J. Regehr, B.P. Casavant and D.J. Beebe, J. Lab Autom., 2013, 18, 206-11; S.M. Berry, E.N. Chin, S.S. Jackson, L.N. Strotman, M. Goel, N.E. Thompson, et al., Anal Biochem., 2014, 447, 133.

19 S.M. Berry, L. J. Maccoux, D. J. Beebe, Anal. Chem. 2012, 84, 5518; S. M. Berry, E. T. Alarid and D. J. Beebe, Lab Chip 2011, 11, 1747.

20 S.M. Berry, A.J. LaVanway, H.M. Pezzi, D.J. Guckenberger, M.A. Anderson, J.M. Loeb, et al., J. Mol. Diagn., 2014, 16, 297.

21 O. Mosley, L. Melling, M. D. Tarn, C. Kemp, M. M. N. Esfahani, N. Pamme and K. Shaw, Lab Chip 2016, 16, 2108.

22 B. Ngamsom, A. Truyts, L. Fourie, S. Kumar, M.D. Tarn, A. Iles, K.N. Land and N. Pamme, Chem.: Eur. J. 2017, 23, 12754.

23 L. Marle, G. M. Greenway, Anal Chim Acta 2005, 548, 20.

24 C.M. Dundas, D. Demont and S. Park, Appl. Microbiol. Biotechnol., 2013, 97, 9343.

25 V.S. Forsyth, C.E. Armbruster, S.N. Smith, A. Pirani, A.C. Springman and M.S. Walters, MBio., 2018, 9, e00186.

26 R.R. Muder, C.B. John, D. Rihs, M. M. Wagener, A. Obman, J.E. Stout and V.L. Yu., Clin. Infect. Dis., 2019, 42, 46.

27 L.M. Ndamason, W.J. Marbou and V. Kuete, Afr. Health Sci. 2019, 19, 1525.

28 R.L. Cook, S.L. Hutchison, L. Ostergaard, R.S. Braithwaite and R.B. Ness, Ann. Intern. Med. 2005,142, 914. 